\title{
A Search for Charged Massive Long-lived Particles at $\sqrt{s}=1.96 \mathrm{TeV}$
}

\section{Sudeshna Banerjee ${ }^{* \dagger}$}

Tata Institute of Fundamental Research

E-mail: sudeshna.banerjee@cern.ch

\section{D0 Collaboration, Fermi National Laboratory}

Results will be presented on a search for charged massive long-lived particles (CMLLPs) that are pair produced in p-pbar collisions at $\sqrt{s}=1.96 \mathrm{TeV}$ at the D0 experiment at the Fermilab Tevatron collider. This result is a combination of two searches where either one or both CMLLPs are reconstructed in the detector. Events are selected with muon-like particles that have both speed and ionization energy loss $\mathrm{d} E / \mathrm{d} x$ different from muons produced in p-pbar collisions. In the absence of evidence for CMLLPs in $6.3 \mathrm{fb}^{-1}$ of integrated luminosity, limits are set on the CMLLP masses in several supersymmetric models, excluding masses below $278 \mathrm{GeV}$ for longlived gaugino-like charginos, and masses below $244 \mathrm{GeV}$ for long-lived higgsino-like charginos at the $95 \%$ C.L. Limits are also set on the cross section for pair production of long-lived scalar tau leptons that range from $0.04 \mathrm{pb}$ to $0.008 \mathrm{pb}$ for scalar tau lepton masses of 100 to $300 \mathrm{GeV}$.

The European Physical Society Conference on High Energy Physics -EPS-HEP2013

18-24 July 2013

Stockholm, Sweden

* Speaker.

${ }^{\dagger}$ A footnote may follow. 


\section{Search for CMLLPs}

Several extensions of the standard model (SM) including some SUSY models predict the existence of charged massive long-lived particles (CMLLP) [1]. CMLLPs could have color or electric charge. They may appear as R-hadrons (bound states of squarks or gluinos with SM quarks), as sleptons, or as charginos. They are relatively slow moving at the collision energy of $\sqrt{s}=1.96 \mathrm{TeV}$ and for masses of $100 \mathrm{GeV}$ or greater and have large ionization energy losses $(\mathrm{d} E / \mathrm{d} x)$ due to their slow speeds. These characteristics are different from other particles studied at high energy colliders, and thus their identification is simplified by the corresponding small amount of background. In this study "long-lived" refers to particles that traverse the entire detector before decaying.

Searches for CMLLPs were performed previously by the D0, CDF, CMS, and ATLAS collaborations $[2,4]$. Results on CMLLPs are presented here by combining a search for pair produced CMLLPs performed with $1.1 \mathrm{fb}^{-1}$ integrated luminosity and an analysis based on $5.2 \mathrm{fb}^{-1}$ integrated luminosity. The second analysis includes searches for either a pair of CMLLPs or a single CMLLP signature in an event. To avoid double counting of events, the datasets used for the two search strategies are made statistically independent by removing the events that have been selected for the search of CMLLP pairs from the dataset used to search for single CMLLPs. The D0 detector, which is used for these searches is described in detail in [3].

Signal samples with direct production of a pair of CMLLPs of masses ranging from $100 \mathrm{GeV}$ to $300 \mathrm{GeV}$ are simulated using pythia [5] in combination with several theoretical models as described in [8]. Any source of muons that are not measured correctly contributes to the background. In the search for a pair of CMLLPs $Z \rightarrow \mu \mu$ is the dominant source which is modeled with one million events containing muons from simulated decays of $Z$ bosons using pythia. The dominant background in the single CMLLP search is muons from the decays of $\mathrm{W}$ bosons, which is modeled with data. CMLLPs travel through the D0 detector similar to very heavy, charged muons. Therefore, the variables that are most useful in distinguishing between signal and background are speed $(\beta)$, speed significance $\left(\frac{1-\langle\beta\rangle}{\sigma_{\langle\beta\rangle}}\right), \mathrm{d} E / \mathrm{d} x$ and $\mathrm{d} E / \mathrm{d} x$ significance $\left(\frac{\mathrm{d} E / \mathrm{d} x-1}{\sigma(\mathrm{d} E / \mathrm{d} x)_{N_{c}}}\right)$ where $N_{c}$ is the number of clusters on a track in the Silicon Microstrip Tracker, and $\sigma(d E / d x)_{N_{c}}$ is the spread in the $\mathrm{d} E / \mathrm{d} x$ distribution (which is a function of $N_{c}$ ). Figure 1 shows distributions of these four variables for the highest $p_{T}$ muon in the event. A Boosted Decision Tree (BDT), as implemented in [6], is used to further discriminate signal events from background events.

The dominant values of systematic errors due to various sources, e.g., muon $p_{T}$ resolution, $\mathrm{d} E / \mathrm{d} x$ measurement, background normalization are of the order of $2 \%$ for signal. The main sources for background are $p_{T}$ resolution (11\%) and time silumation (9.5\%) for pair CMLLP search and background normalization (7.2\%) for single CMLLP search.

\section{Results}

In the absence of observed signal, 95\% C.L. limits are placed using a CLs method [7] on masses of CMLLPs as $278 \mathrm{GeV}$ for gaugino-like charginos and $244 \mathrm{GeV}$ for higgsino-like charginos. For stau leptons an upper limit of $0.04 \mathrm{pb}$ to $0.008 \mathrm{pb}$ is set on the production cross section for the mass range of 100 to $300 \mathrm{GeV}$. Combined luminosity used to obtain these results is $6.3 \mathrm{fb}^{-1}$. Top squarks with masses below $285 \mathrm{GeV}$ are excluded at $95 \%$ C.L. with a charge flipping 

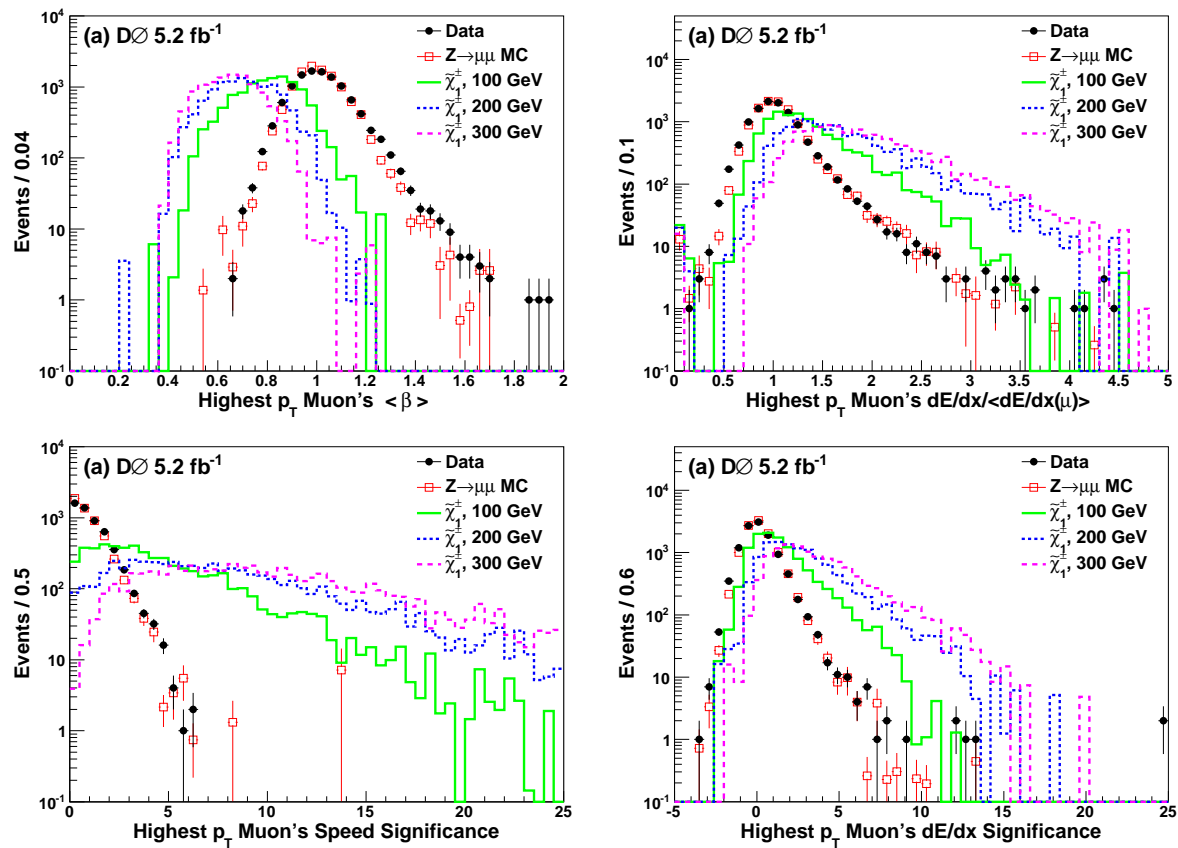

Figure 1: (color online) Distributions of (a) $\beta$ (b) $d E / d x$ (c) speed significance and (d) $d E / d x$ significance of the highest pT muon in the event for charginos of masses $100 \mathrm{GeV}, 200 \mathrm{GeV}$ and $300 \mathrm{GeV}$, data and $Z \rightarrow \mu \mu \mathrm{MC}$ events. The distributions are normalized to the same number of events.

probability of $38 \%$ with $5.2 \mathrm{fb}^{-1}$ of data. [8]. Figure 2 (a) and (b) show typical BDT distributions for the pair and the single CMLLP search respectively. Figure 2 (c) and (d) show the limits for gaugino-like and higgsino-like charginos respectively.

\section{References}

[1] M. Byrne et al. Phys. Rev. D 66, 075007 (2002), M. Fairbairn et al., Phys. Rept. 438, 1 (2007), R. Mackeprang, CERN-THESIS-2007-109, R. Mackeprang et al., Eur. Phys. J. C66, 493 (2010).

[2] D. Acosta et al. (CDF Collaboration), Phys. Rev. Lett. 103, 021802 (2009), V. Khachatryan et al. (CMS Collaboration), J. High Energy Phys. 03, 024 (2011), G. Aad et al. (ATLAS Collaboration), J. High Energy Phys. 03, 024 (2011).

[3] V. M. Abazov et al. (D0 Collaboration), Nucl. Instrum. Methods Phys. Res., Sect. A 565, 463 (2006); M. Abolins et al., R. Angstadt et al., Nucl. Instrum. Methods Phys. Res., Sect. A 622, 298 (2010).

[4] V. Abazov et al. (D0 Collaboration), Phys. Rev. Lett. 102, 161802 (2009), V. Abazov et al. (D0 Collaboration), Phys. Rev. Lett. 108, 121802 (2012).

[5] T. Sjostrand, S. Mrenna, and P. Skands, J. High Energy Phys. 05 (2006) 026; we use version 6.409.

[6] A. Hoecker et al., PoS ACAT 040 (2007), arXiv:physics/0703039.

[7] T. Junk, Nucl. Instrum. Methods Phys. Res. A 434, 435(1999), A. Read, J. Phys. G 28, 2693 (2002), W. Fisher, FERMILAB-TM-2386-E (2006).

[8] V. M. Abazov et al. (D0 Collaboration), Phys. Rev. D 87, 052011 (2013). 

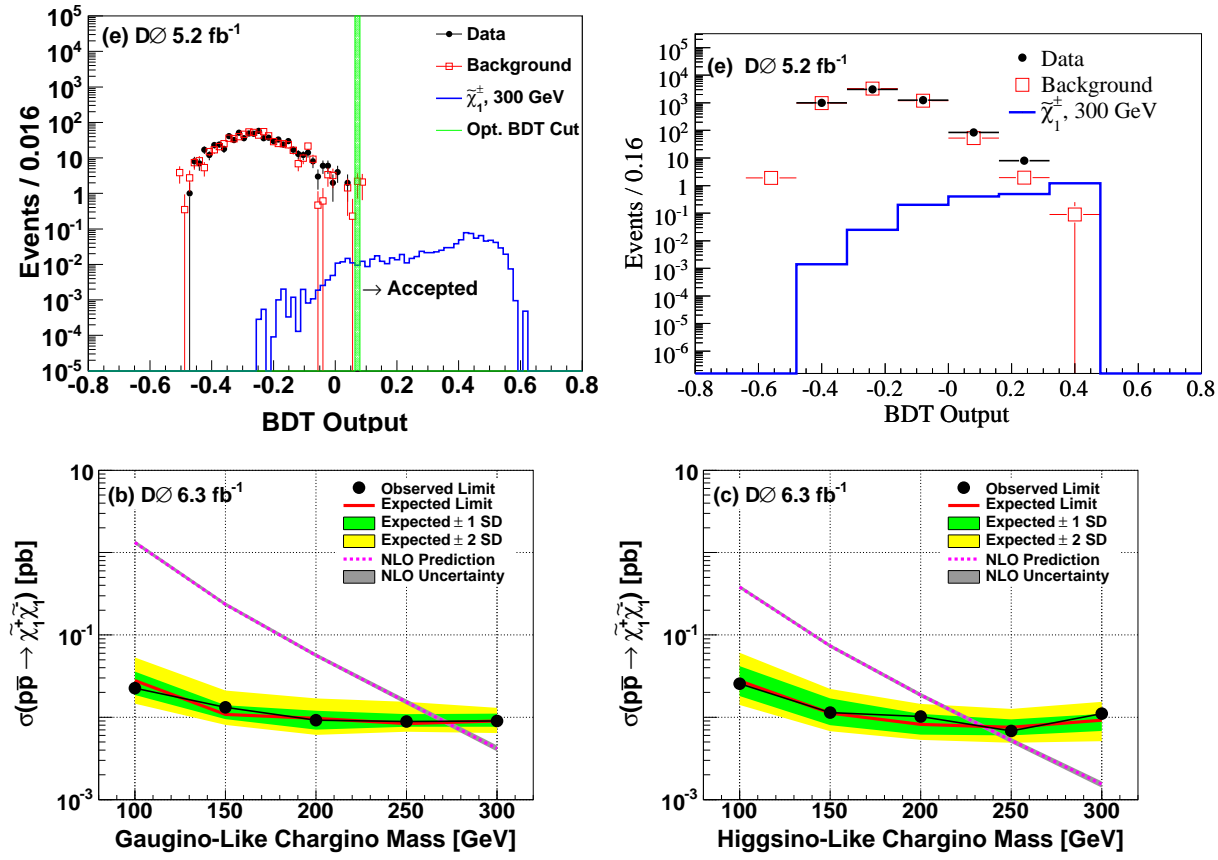

Figure 2: (color online) BDT-output distribution for simulated gaugino-like char gino of $300 \mathrm{GeV}$ mass for the search for a (a) CMLLP pair (selection requirement on the BDT value is shown with a green vertical line), (b) single CMLLPs. Comb ined limits at 95\% C.L. on production cross sections of a pair of gauginolike charginos, and higgsino-like charginos as a function of their masses with $6.3 \mathrm{fb}^{-1}$ of data collected by the D0 experiment. 